\title{
RADAU QUADRATURE FOR AN ALMOST QUASI-HERMITE-FEJÉR-TYPE INTERPOLATION IN RATIONAL SPACES
}

\author{
SHRAWAN KUMAR ${ }^{1}$, NEHA MATHUR ${ }^{2}$, \\ VISHNU NARAYAN MISHRA ${ }^{3, *}$, PANKAJ MATHUR ${ }^{1, *}$ \\ ${ }^{1}$ Department of Mathematics and Astronomy, University of Lucknow, Lucknow \\ ${ }^{2}$ Department of Mathematics, Career Convent Degree College, Lucknow \\ ${ }^{3}$ Department of Mathematics, Indira Gandhi National Tribal University, \\ Lalpur, Amarkantak, Anuppur, Madhya Pradesh 484 887, India
}

\begin{abstract}
*Corresponding authors: vishnunarayanmishra@gmail.com, pankaj_mathur14@yahoo.co.in Abstract. In this paper, we have studied an almost quasi Hermite-Fejér-type interpolation in rational spaces. A Radau type quadrature formula has also been obtained for the same.
\end{abstract}

\section{Introduction}

Hermite Fejér and Quasi-Hermite-Fejér-type interpolation processes has been a subject of interest for several mathematicians. In almost all the cases the interpolatory polynomials are considered on the nodes which are the zeros of certain classical orthogonal polynomials. The main idea of the present paper is to construct a rational interpolation process and its corresponding quadrature formula with prescribed nodes based on the Chebyshev Markov fractions.

Received November $1^{\text {st }}, 2019$; accepted March $2^{\text {nd }}, 2020$; published February $1^{\text {st }}, 2021$.

2010 Mathematics Subject Classification. Primary 05C38, 15A15; Secondary 05A15, 15A18.

Key words and phrases. Almost Quasi-Hermite-Fejér-type interpolation; Radau-type quadrature; rational space; prescribed poles; Chebyshev-Markov fractions.

(C)2021 Authors retain the copyrights of their papers, and all open access articles are distributed under the terms of the Creative Commons Attribution License. 
Chebyshev and Markov introduced rational cosine and sine fractions [3] which generalizes Chebyshev polynomials, possesses many similar properties $([2,10,11])$ and are called Chebychev-Markov rational fractions. Different aspects of the rational generalization of Chebyshev polynomials are discussed in many works $([1,12])$.

In 1962, Rusak [9] initiated the study of interpolation processes by means of rational functions on the interval $[-1,1]$. The nodes were taken to be the zeros of Chebyshev-Markov rational fractions. In [6] rational interpolation functions of Hermite-Fejér-type were constructed [7]. Min [4] was the first to consider the rational quasi-Hermite-type interpolation. He constructed the interpolatory function and proved its uniform convergence for the continuous functions on the segment with the restriction that the poles of the approximating rational functions should not have limit points on the interval $[-1,1]$.

Recently, based on the ideas of [6] and using method that was different from that of [4], Rouba et. al. ( [5], [8]) revisited the rational interpolation functions of Hermite-Fejér-type. They also proved the uniform convergence of the interpolation process for the function $f \in C[-1,1]$ and obtained explicitly its corresponding Lobatto type quadrature formula.

In this paper, we have considered an almost quasi-Hermite-Fejér-type interpolation process on the zeros of the rational Chebyshev-Markov sine fraction on the semi closed interval $(-1,1]$, that is, when the interpolatory condition is prescribed only at one of the end points. A Radau type quadrature formula corresponding to the interpolation process has also been obtained.

\section{Preliminaries}

Consider a set of points $a_{k}, k=0,1, \cdots, 2 n-1$ which are either real and $a_{k} \in(-1,1)$ or be paired by complex conjugation. Also let $U_{n}(x)$ be the rational Chebyshev-Markov sine fraction,

$$
U_{n}(x)=\frac{\sin \mu_{2 n}(x)}{\sqrt{1-x^{2}}}
$$

where,

$$
\begin{gathered}
\mu_{2 n}(x)=\frac{1}{2} \sum_{k=0}^{2 n-1} \arccos \frac{x+a_{k}}{1+a_{k} x}, \\
\mu_{2 n}^{\prime}(x)=-\frac{\lambda_{2 n}(x)}{\sqrt{1-x^{2}}}, \\
\lambda_{2 n}(x)=\frac{1}{2} \sum_{k=0}^{2 n-1} \frac{\sqrt{1-a_{k}^{2}}}{1+a_{k} x}, \quad n \in N .
\end{gathered}
$$

Also

$$
U_{n}^{\prime}(x)=\frac{-\cos \mu_{2 n}(x) \lambda_{2 n}(x) \sqrt{1-x^{2}}+x \sin \mu_{2 n}(x)}{\left(1-x^{2}\right)^{3 / 2}}
$$


and

$$
U_{n}^{\prime}\left(x_{k}\right)=-\frac{\lambda_{2 n}\left(x_{k}\right)}{\left(1-x_{k}^{2}\right)}
$$

Let $\mathcal{R}_{2 n-1}\left(a_{0}, a_{1}, \cdots, a_{2 n-1}\right)$ be a rational space defined as

$$
\mathcal{R}_{2 n-1}\left(a_{0}, a_{1}, \cdots, a_{2 n-1}\right):=\left\{\frac{p_{2 n-1}(x)}{\prod_{k=0}^{2 n-1}\left(1+a_{k} x\right)}\right\}
$$

where $p_{2 n-1}(x)$ is a polynomial of degree $\leq 2 n-1$ and $\left\{a_{k}\right\}_{k=0}^{2 n-1}$ are real and belong to $[-1,1]$ or are paired by complex conjugation.

The rational fraction $U_{n}(x)$ can be expressed as

$$
U_{n}(x)=\frac{P_{n-1}(x)}{\sqrt{\Pi_{k=0}^{2 n-1}\left(1+a_{k} x\right)}}
$$

where $P_{n-1}(x)$ is an algebraic polynomial of degree $n-1$ with real coefficient. The fraction $U_{n}(x)$ has $n-1$ zeros on the interval $(-1,1)$ given by,

$$
-1<x_{n-1}<x_{n-2}<\cdots<x_{2}<x_{1}<1, \quad \mu_{2 n}\left(x_{k}\right)=k \pi, k=1,2, \cdots, n-1,
$$

where $\mu_{2 n}(x)$ is given by (2.2). Also, the rational function $\lambda_{2 n}(x)$, given by (2.4), can be expressed as

$$
\lambda_{2 n}(x)=\frac{q_{2 n-1}(x)}{\prod_{k=0}^{2 n-1}\left(1+a_{k} x\right)}
$$

where $q_{2 n-1}(x)$ is a polynomial of degree atmost $2 n-1$. It has no zeros on $[-1,1]$.

\section{Almost Quasi-Hermite-FeJÉR-Type interpolation}

Let $x_{0}=1$. Then for any function $f \in C(-1,1]$ the almost quasi type Hermite interpolation function $H_{n}(x, f)$ satisfying the conditions

$$
\begin{aligned}
& H_{n}\left(x_{k}, f\right)=f\left(x_{k}\right), \quad k=0,1, \cdots, n-1, \\
& H_{n}^{\prime}\left(x_{k}, f\right)=y_{k}, \quad k=1,2, \cdots, n-1,
\end{aligned}
$$

is given by

$$
H_{n}(x, f)=\sum_{k=0}^{n-1} f\left(x_{k}\right) A_{k}(x)+\sum_{k=1}^{n-1} y_{k} B_{k}(x),
$$

where $y_{k}, k=1,2, \cdots, n-1$ are arbitrarily given real numbers, $\left\{A_{k}(x)\right\}_{k=0}^{n-1}$ and $\left\{B_{k}(x)\right\}_{k=1}^{n-1}$ are fundamental functions satisfying the conditions

$$
\left\{\begin{array}{l}
A_{k}\left(x_{j}\right)=\delta_{k j}, \quad j, k=0,1, \cdots, n-1, \\
A_{k}^{\prime}\left(x_{j}\right)=0, \quad j=1,2, \cdots, n-1, k=0,1, \cdots, n-1
\end{array}\right.
$$


and

$$
\left\{\begin{array}{l}
B_{k}\left(x_{j}\right)=0, \quad j=0,1, \cdots, n-1, k=1,2, \cdots, n-1, \\
B_{k}^{\prime}\left(x_{j}\right)=\delta_{k j}, \quad j, k=1,2, \cdots, n-1 .
\end{array}\right.
$$

\section{Explicit Representation of the Fundamental Functions}

Lemma 4.1. The fundamental functions $\left\{A_{k}(x)\right\}_{k=0}^{n-1}$ satisfying the conditions (3.4) can be explicitly represented as for $k=1,2, \cdots, n-1$

$$
A_{k}(x)=\frac{\left(1+x_{k}\right)\left(1-x_{k}^{2}\right)(1-x)\left\{1-b_{k}\left(x-x_{k}\right)\right\} U_{n}^{2}(x)}{\lambda_{2 n}\left(x_{k}\right)\left(x-x_{k}\right)^{2} \lambda_{2 n}(x)}
$$

where

$$
b_{k}=\frac{2 x_{k}-1}{1-x_{k}^{2}}
$$

and

$$
A_{0}(x)=\frac{U_{n}^{2}(x)}{\lambda_{2 n}(x) \lambda_{2 n}(1)} .
$$

Proof. We will show that $A_{k}(x), k=0,1, \cdots, n-1$ defined by (4.1) and (4.3) satisfy the conditions (3.4) Obviously for $k=1,2, \cdots, n-1, A_{k}\left(x_{0}\right)=0$ and $A_{k}\left(x_{j}\right)=0, j=1,2, \cdots, n-1$ when $j \neq k$. For $j=k$ using the l'Hospital's rule, we have

$$
\begin{aligned}
\lim _{x \rightarrow x_{k}} A_{k}(x) & =\frac{\left(1-x_{k}^{2}\right)}{\lambda_{2 n}^{2}\left(x_{k}\right)}\left(\lim _{x \rightarrow x_{k}} \frac{\sin \mu_{2 n}(x)}{\left(x-x_{k}\right)}\right)^{2} \\
& =\frac{\left(1-x_{k}^{2}\right)}{\lambda_{2 n}^{2}\left(x_{k}\right)}\left(\lim _{x \rightarrow x_{k}} \frac{-\lambda_{2 n}(x) \cos \mu_{2 n}(x)}{\sqrt{1-x^{2}}}\right)^{2} \\
& =1 .
\end{aligned}
$$

On differentiating (4.1) with respect $x$ we get

$$
\begin{aligned}
& A_{k}^{\prime}(x)=\frac{\left(1+x_{k}\right)\left(1-x_{k}^{2}\right)}{\lambda_{2 n}\left(x_{k}\right)}\left[\frac{2\left\{1-b_{k}\left(x-x_{k}\right)\right\}}{(1+x) \lambda_{2 n}(x)}\left(\frac{\sin \mu_{2 n}(x)}{x-x_{k}}\right)\left(\frac{\sin \mu_{2 n}}{x-x_{k}}\right)^{\prime}\right. \\
& -\frac{b_{k}(1+x) \lambda_{2 n}(x)+\left\{1-b_{k}\left(x-x_{k}\right)\right\}\left\{(1+x) \lambda_{2 n}^{\prime}(x)+\lambda_{2 n}(x)\right\}}{(1+x)^{2} \lambda_{2 n}^{2}(x)} \\
& \left.\times\left(\frac{\sin \mu_{2 n}(x)}{x-x_{k}}\right)^{2}\right]
\end{aligned}
$$

then for $j \neq k$ we have $A_{k}^{\prime}\left(x_{j}\right)=0, j=1,2, \cdots, n-1$. For $j=k$,

$$
\begin{aligned}
\lim _{x \rightarrow x_{k}} A_{k}^{\prime}(x)= & \frac{\left(1-x_{k}^{2}\right)}{\lambda_{2 n}^{2}\left(x_{k}\right)}\left[2 \lim _{x \rightarrow x_{k}}\left(\left(\frac{\sin \mu_{2 n}(x)}{x-x_{k}}\right)\left(\frac{\sin \mu_{2 n}(x)}{x-x_{k}}\right)^{\prime}\right)\right. \\
& -\frac{b_{k}\left(1+x_{k}\right) \lambda_{2 n}\left(x_{k}\right)+\left(1+x_{k}\right) \lambda_{2 n}^{\prime}\left(x_{k}\right)+\lambda_{2 n}\left(x_{k}\right)}{\left(1+x_{k}\right) \lambda_{2 n}\left(x_{k}\right)} \\
& \left.\times\left(\lim _{x \rightarrow x_{k}} \frac{\sin \mu_{2 n}(x)}{x-x_{k}}\right)^{2}\right]
\end{aligned}
$$


We know that

$$
\lim _{x \rightarrow x_{k}} \frac{\sin \mu_{2 n}(x)}{\left(x-x_{k}\right)}=\mu_{2 n}^{\prime}\left(x_{k}\right) \cos \mu_{2 n}\left(x_{k}\right)=-\frac{\lambda_{2 n}\left(x_{k}\right)}{\sqrt{1-x_{k}^{2}}}
$$

and

$$
\lim _{x \rightarrow x_{k}}\left(\frac{\sin \mu_{2 n}(x)}{x-x_{k}}\right)^{\prime}=\frac{1}{2} \cos \mu_{2 n}\left(x_{k}\right) \mu_{2 n}^{\prime \prime}\left(x_{k}\right)
$$

where

$$
\mu_{2 n}^{\prime \prime}(x)=-\frac{\left(1-x^{2}\right) \lambda_{2 n}^{\prime}(x)+x \lambda_{2 n}(x)}{\left(1-x^{2}\right)^{3 / 2}}
$$

then

$$
\lim _{x \rightarrow x_{k}} A_{k}^{\prime}(x)=\left[\frac{2 x_{k}-1}{\left(1-x_{k}^{2}\right)}-b_{k}\right]=0
$$

due to (4.2) which shows that $A_{k}(x), k=1,2, \cdots, n-1$, given by (4.1), satisfy all the conditions given by (3.4).

Similarly, for $A_{0}(x)$, given by (4.3), we have that $A_{0}\left(x_{j}\right)=0, j=1, \cdots, n-1$. For $j=0$ and using the fact that $U_{n}(1)=\lambda_{2 n}(1)$, we have $A_{0}\left(x_{0}\right)=1$. Again by differentiating (4.3) with respect $x$, we get $A_{0}^{\prime}\left(x_{j}\right)=0, j=1,2, \cdots, n-1$. Thus $A_{0}(x)$ given by (4.3) satisfy the conditions (3.4) for $j=0$, which completes the proof of the lemma.

Lemma 4.2. The fundamental functions $\left\{B_{k}(x)\right\}_{k=1}^{n-1}$ satisfying the conditions (3.5) can be explicitly represented as

$$
B_{k}(x)=\frac{(1-x)\left(1+x_{k}\right)\left(1-x_{k}^{2}\right) U_{n}^{2}(x)}{\lambda_{2 n}(x) \lambda_{2 n}\left(x_{k}\right)\left(x-x_{k}\right)} .
$$

Proof. Obviously, $B_{k}\left(x_{j}\right)=0, k=1,2, \cdots, n-1, j=0,1, \cdots, n-1$ and for $j \neq k, B_{k}^{\prime}\left(x_{j}\right)=0, j, k=$ $1,2, \cdots, n-1$. For $j=k$,

$$
\begin{aligned}
\lim _{x \rightarrow x_{k}} B_{k}^{\prime}(x) & =\frac{\left(1-x_{k}^{2}\right)^{2}}{\lambda_{2 n}^{2}\left(x_{k}\right)} \lim _{x \rightarrow x_{k}}\left(\frac{U_{n}^{2}(x)}{\left(x-x_{k}\right)}\right) \\
& =\frac{\left(1-x_{k}^{2}\right)^{2}}{\lambda_{2 n}^{2}\left(x_{k}\right)} \lim _{x \rightarrow x_{k}}\left(\frac{2 U_{n}(x) U_{n}^{\prime}(x)}{\left(x-x_{k}\right)}-\left(\frac{U_{n}(x)}{x-x_{k}}\right)^{2}\right) \\
& =\frac{\left(1-x_{k}^{2}\right)^{2}}{\lambda_{2 n}^{2}\left(x_{k}\right)}\left(U_{n}^{\prime}\left(x_{k}\right)\right)^{2} \\
& =1,
\end{aligned}
$$

due to (2.6), which proves that $B_{k}(x), k=1,2, \cdots, n-1$ given by (4.7) satisfy all the conditions given by (3.5).

From Lemma 4.1 and Lemma 4.2 it follows that $H_{n}(f, x)$ satisfying the conditions (3.1) is an almost quasi Hermite interpolation. 
Theorem 4.1. The function $H_{n}(f, x)$ is a rational function of degree atmost $2 n-1$ that is

$$
H_{n}(f, x) \in \mathcal{R}_{2 n-1}\left(a_{0}, a_{1}, \cdots, a_{2 n-1}\right) .
$$

Proof. Since $U_{n} \in \mathcal{P}_{n-1}\left(a_{0}, a_{1}, \cdots, a_{2 n-1}\right)$, we can express it as

$$
U_{n}(x):=\frac{S_{n-1}(x)}{\left(S_{n}^{*}(x)\right)^{1 / 2}}
$$

where $S_{n}^{*}(x):=\left(x-a_{0}\right)\left(x-a_{1}\right) \cdots\left(x-a_{2 n-1}\right), S_{n-1}(x):=c_{n-1}\left(x-x_{1}\right)\left(x-x_{2}\right) \cdots\left(x-x_{n-1}\right)$ and $c_{n-1}$ depends on $\mathrm{n}$ and $\left\{a_{k}\right\}_{k=0}^{2 n-1}$. So, we have

$$
\ell_{k}(x)=\left(\frac{S_{n}^{*}\left(x_{k}\right)}{S_{n}^{*}(x)}\right)^{1 / 2} q_{k}(x), \quad k=1,2, \cdots, n-1,
$$

where

$$
q_{k}(x):=\frac{S_{n-1}(x)}{S_{n-1}^{\prime}\left(x_{k}\right)\left(x-x_{k}\right)}, \quad k=1,2, \cdots, n-1 .
$$

Thus, $\ell_{k}(x) \in \mathcal{P}_{n-1}\left(a_{0}, a_{1}, \cdots, a_{2 n-1}\right)$, thus by (3.3), (4.1) and (4.7) we easily find that

$$
H_{n}(f, x)=\frac{t_{2 n-1}(x)}{q_{2 n-1}(x)}
$$

where $t_{2 n-1}(x)$ is a polynomial of degree $\leq 2 n-1$, which proves the lemma.

Let $y_{k}=0, k=1,2, \cdots, n-1$ then (3.3) reduces to

$$
H_{n}(f, x)=\sum_{k=1}^{n-1} f\left(x_{k}\right) A_{k}(x)+f(1) A_{0}(x)
$$

which is an almost quasi Hermite Fejér interpolation function for $f \in C[-1,1]$.

\section{RADAU-TYPE QUADRATURE FORMUla}

For a given function $f$ defined on $[-1,1]$, we define the function

$$
G_{n}(x, f)=\sum_{k=0}^{n-1} f\left(x_{k}\right) h_{k}(x)
$$

where,

$$
\begin{array}{r}
h_{k}(x)=\frac{1-x}{1-x_{k}}\left[1-\left(\frac{U_{n}^{\prime \prime}\left(x_{k}\right)}{U_{n}^{\prime}\left(x_{k}\right)}-\frac{1}{\left(1-x_{k}\right)}\right)\left(x-x_{k}\right)\right] \ell_{k}^{2}(x), \\
k=1,2, \cdots, n-1
\end{array}
$$

and

$$
h_{0}(x)=\frac{U_{n}^{2}(x)}{U_{n}^{2}(1)}
$$


We have that $G_{n}(f, x) \in \mathcal{R}_{2 n-1}\left(a_{0}, a_{1}, \cdots, a_{2 n-1}\right)$. Also the rational function $G_{n}(f, x)$ is an almost quasi Hermite Fejér interpolation function. Let

$$
A_{k}=\int_{-1}^{1} \frac{1}{\sqrt{1-x^{2}}} h_{k}(x) d x, \quad k=1,2, \cdots, n-1
$$

and

$$
A_{0}=\int_{-1}^{1} \frac{1}{\sqrt{1-x^{2}}} \frac{U_{n}^{2}(x)}{U_{n}^{2}(1)} d x
$$

then the Radau-type quadrature formula is given by

$$
\int_{-1}^{1} \frac{f(x)}{\sqrt{1-x^{2}}} d x=A_{0} f(1)+\sum_{k=1}^{n-1} A_{k} f\left(x_{k}\right)
$$

With respect to this quadrature formula, we have the following

Theorem 5.1. The quadrature formula (5.4) can be expressed as

$$
\int_{-1}^{1} \frac{f(x)}{\sqrt{1-x^{2}}} d x=\frac{2 \pi}{\lambda_{2 n}(1)} f(1)+\sum_{k=1}^{n-1} \frac{\pi}{\lambda_{2 n}\left(x_{k}\right)} f\left(x_{k}\right) .
$$

Lemma 5.1. For $k=1,2, \cdots, n-1$,

$$
\int_{-1}^{1}(1-x)\left(x-x_{k}\right) \ell_{k}^{2}(x) d x=0 .
$$

Proof. We have that for $k=1,2, \cdots, n-1$,

$$
\begin{aligned}
\ell_{k}^{2}(x) & =\frac{U_{n}^{2}(x)}{\left(U_{n}^{\prime}\right)^{2}\left(x_{k}\right)\left(x-x_{k}\right)^{2}} \\
& =\frac{\left(1-x_{k}^{2}\right)^{2} \sin ^{2} \mu_{2 n}(x)}{\lambda_{2 n}^{2}\left(x_{k}\right)\left(1-x^{2}\right)\left(x-x_{k}\right)^{2}} .
\end{aligned}
$$

Also,

$$
U_{n}(1)=\lim _{x \rightarrow 1} \frac{\sin \mu_{2 n}(x)}{\sqrt{1-x^{2}}}=\lambda_{2 n}(1)
$$

and

$$
U_{n}(-1)=(-1)^{n+1} \lambda_{2 n}(-1)
$$

By these equalities, the left hand side of (5.6) can be represented as

$$
I_{k}=\int_{-1}^{1} \frac{\sin ^{2} \mu_{2 n}(x)}{(1+x) \sqrt{1-x^{2}}\left(x-x_{k}\right)} d x
$$

Consider the transformation

$$
x=\frac{1-y^{2}}{1+y^{2}}
$$

which gives

$$
d x=-\frac{4 y}{\left(1+y^{2}\right)^{2}} d y
$$




$$
\begin{gathered}
x-x_{k}=-\frac{2\left(y^{2}-y_{k}^{2}\right)}{\left(1+y^{2}\right)\left(1+y_{k}^{2}\right)}, \\
1+x=\frac{2}{1+y^{2}}, \\
\sqrt{1-x^{2}}=\frac{2 y}{1+y^{2}} .
\end{gathered}
$$

We know that,

$$
\sin \mu_{2 n}\left(\frac{1-y^{2}}{1+y^{2}}\right)=\sin \phi_{2 n}(y)
$$

where $\sin \phi_{2 n}(y)$ is a Bernstein sine fraction

$$
\sin \phi_{2 n}(y)=\frac{1}{2 i}\left(\chi_{n}(y)-\chi_{n}^{-1}(y)\right)
$$

where $\chi_{n}(y)=\prod_{j=0}^{2 n-1} \frac{y-z_{j}}{y-z_{j}}$ and $z_{k}$ are the roots of the equations $y^{2}+\left(1+a_{k}\right)\left(1-a_{k}\right)^{-1}=0, \mathcal{I} z_{k}>0, k=$ $0,1, \cdots, 2 n-1$. Taking into account the assumptions on the parameters $a_{k}, k=0,1, \cdots, 2 n-1$, we have the following: 1) $\left.z_{0}=i, 2\right)$ if $a_{k}$ and $a_{l}$ are paired by complex conjugation, then the corresponding numbers $z_{k}$ and $z_{l}$ are symmetric with respect to the imaginary axis. Besides, the function $\sin \phi_{2 n}(y)$ has zeros at $\pm y_{k}, y_{k}=\sqrt{\left(1-x_{k}\right) /\left(1+x_{k}\right)}, k=1,2, \cdots, n-1$. Thus,

$$
\begin{aligned}
I_{k} & =-\frac{1+y_{k}^{2}}{4} \int_{-\infty}^{\infty} \frac{\left(1+y^{2}\right) \sin ^{2} \phi_{2 n}(y)}{y^{2}-y_{k}^{2}} d y \\
& =-\frac{1+y_{k}^{2}}{4} \lim _{z \rightarrow y_{k}, \mathcal{I} z_{k}>0} J_{k}(z)
\end{aligned}
$$

where

$$
J_{k}(z)=\int_{-\infty}^{\infty} \frac{\left(1+y^{2}\right) \sin ^{2} \phi_{2 n}(y)}{y^{2}-z^{2}} d y
$$

From (5.17) we get

$$
\sin ^{2} \phi_{2 n}(y)=-\frac{1}{4}\left(\chi_{n}^{2}(y)-2+\chi_{n}^{-2}(y)\right)
$$

due to which, we have

$$
J_{k}(z)=-\frac{1}{4}\left(J_{k 1}(z)-2 J_{k 2}(z)+J_{k 3}(z)\right)
$$

where

$$
\begin{gathered}
J_{k 1}(z)=\int_{-\infty}^{\infty} \frac{\left(1+y^{2}\right) \chi_{n}^{2}(y)}{y^{2}-z^{2}} d y \\
J_{k 2}(z)=\int_{-\infty}^{\infty} \frac{\left(1+y^{2}\right)}{y^{2}-z^{2}} d y
\end{gathered}
$$


and

$$
J_{k 3}(z)=\int_{-\infty}^{\infty} \frac{\left(1+y^{2}\right) \chi_{n}^{-2}(y)}{y^{2}-z^{2}} d y
$$

Since $J_{k 1}(z)$ has only singular point $y=z$ in the upper half plane. Thus by the residue theorem we have

$$
\begin{aligned}
J_{k 1}(z) & =2 \pi i \lim _{y \rightarrow z} \frac{\left(1+y^{2}\right) \chi_{n}^{2}(y)}{(y+z)} \\
& =\frac{1+z^{2}}{z} \chi_{n}^{2}(z) \pi i .
\end{aligned}
$$

Similarly,

$$
J_{k 3}(z)=\frac{1+z^{2}}{z} \chi_{n}^{-2}(z) \pi i
$$

Also, $J_{k 2}(z)$ has only singular point $y=z$ in the upper half plane, therefore by the residue theorem, we have

$$
J_{k 2}(z)=2 \pi i \lim _{y \rightarrow z} \frac{\left(1+y^{2}\right)}{(y+z)}=\frac{1+z^{2}}{z} \pi i .
$$

Putting the value of $J_{k 1}(z), J_{k 3}(z)$ and $J_{k 2}(z)$ from (5.22), (5.23) and (5.24) respectively in (5.21) we get

$$
J_{k}(z)=-\frac{1}{4}\left(\frac{1+z^{2}}{z} \chi_{n}^{2}(z) \pi i-2 \frac{1+z^{2}}{z} \pi i+\frac{1+z^{2}}{z} \chi_{n}^{-2}(z) \pi i\right)
$$

which by (5.18) gives

$$
I_{k}=\frac{1+y_{k}^{2}}{16} \lim _{z \rightarrow y_{k}, \mathcal{I}_{k}>0}\left(\frac{1+z^{2}}{z} \chi_{n}^{2}(z) \pi i+\frac{1+z^{2}}{z} \chi_{n}^{-2}(z) \pi i-2 \frac{1+z^{2}}{z} \pi i\right) .
$$

Since $\chi_{n}\left(y_{k}\right)=1$, thus it follows that $I_{k}=0$, which completes the proof of the lemma.

Lemma 5.2. For $k=1,2, \cdots, n-1$,

$$
A_{k}=\frac{\pi}{\lambda_{2 n}\left(x_{k}\right)}
$$

Proof. Due to Lemma 5.1 and by putting the value of $\ell_{k}^{2}(x), k=1,2, \cdots, n-1$, from (5.7) in (5.2), we have

$$
A_{k}=\frac{\left(1+x_{k}\right)\left(1-x_{k}^{2}\right)}{\lambda_{2 n}^{2}\left(x_{k}\right)} \int_{-1}^{1} \frac{\sin ^{2} \mu_{2 n}(x)}{\left(x-x_{k}\right)^{2}(1+x) \sqrt{1-x^{2}}} d x .
$$

We find the integrals

$$
I_{k}^{*}=\int_{-1}^{1} \frac{\sin ^{2} \mu_{2 n}(x)}{\left(x-x_{k}\right)^{2}(1+x) \sqrt{1-x^{2}}} d x, \quad k=1,2, \cdots, n-1,
$$

by using the transformation $(5.11),(5.12),(5.13),(5.14),(5.15)$ and (5.16), we have

$$
I_{k}^{*}=\frac{\left(1+y_{k}^{2}\right)^{2}}{8} \int_{-\infty}^{\infty} \frac{\left(1+y^{2}\right)^{2} \sin ^{2} \phi_{2 n}(y)}{\left(y^{2}-y_{k}^{2}\right)^{2}} d y
$$

Consider the auxiliary integral

$$
J_{k}^{*}(z)=\int_{-\infty}^{\infty} \frac{\left(1+y^{2}\right)^{2} \sin ^{2} \phi_{2 n}(y)}{\left(y^{2}-z^{2}\right)^{2}} d y
$$


then $I_{k}^{*}$ can be written as

$$
I_{k}^{*}=\frac{\left(1+y_{k}^{2}\right)^{2}}{8} \lim _{z \rightarrow y_{k}, \Im z_{k}>0} J_{k}^{*}(z) .
$$

which due to (5.20) can be expressed as

$$
I_{k}^{*}=\frac{\left(1+y_{k}^{2}\right)^{2}}{32} \lim _{z \rightarrow y_{k}, \Im z_{k}>0}\left(I_{1 k}^{*}(z)-2 I_{2 k}^{*}(z)+I_{3 k}^{*}(z)\right)
$$

where,

$$
\begin{gathered}
I_{1 k}^{*}(z)=\int_{-\infty}^{\infty} \frac{\left(1+y^{2}\right)^{2}}{\left(y^{2}-z^{2}\right)^{2}} \chi_{n}^{2}(y) d y \\
I_{2 k}^{*}(z)=\int_{-\infty}^{\infty} \frac{\left(1+y^{2}\right)^{2}}{\left(y^{2}-z^{2}\right)^{2}} d y
\end{gathered}
$$

and

$$
I_{3 k}^{*}(z)=\int_{-\infty}^{\infty} \frac{\left(1+y^{2}\right)^{2}}{\left(y^{2}-z^{2}\right)^{2}} \chi_{n}^{-2}(y) d y .
$$

Since $z_{0}=i$ the integrand of $I_{1 k}^{*}(z)$ has only singular point $y=z$ in the upper half plane. Thus by the residue theorem, we have

$$
\begin{aligned}
I_{1 k}^{*}(z) & =2 \pi i \lim _{y \rightarrow z} \frac{d}{d y}\left(\frac{\left(1+y^{2}\right)^{2}}{(y+z)^{2}} \chi_{n}^{2}(y)\right) \\
& =2 \pi i \lim _{y \rightarrow z}\left[\chi_{n}^{2}(y) \frac{d}{d y} \frac{\left(y^{2}+1\right)^{2}}{(y+z)^{2}}+\frac{\left(y^{2}+1\right)^{2}}{(y+z)^{2}} \frac{d}{d y} \chi_{n}^{2}(y)\right] .
\end{aligned}
$$

Since,

$$
\chi_{n}(y)=\prod_{j=0}^{2 n-1} \frac{y-z_{j}}{y-\bar{z}_{j}}
$$

which by logarithmic differentiation gives

$$
\frac{d}{d y} \chi_{n}(y)=\chi_{n}(y) \sum_{j=0}^{2 n-1} \frac{z_{j}-\bar{z}_{j}}{\left(y-z_{j}\right)\left(y-\bar{z}_{j}\right)} .
$$

Also,

$$
\frac{d}{d y}\left(\frac{\left(y^{2}+1\right)^{2}}{(y+z)^{2}}\right)=\frac{2 y^{4}+4 y^{3} z+4 y z-2}{(y+z)^{3}}
$$

Therefore,

$$
\begin{aligned}
I_{1 k}^{*}(z)= & 2 \pi i \chi_{n}^{2}(z)\left[\frac{3 z^{4}+2 z^{2}-1}{4 z^{3}}\right. \\
& \left.+\frac{\left(z^{2}+1\right)^{2}}{2 z^{2}} \sum_{j=0}^{2 n-1} \frac{z_{j}-\bar{z}_{j}}{\left(z-z_{j}\right)\left(z-\bar{z}_{j}\right)}\right] .
\end{aligned}
$$

Similarly,

$$
\begin{aligned}
I_{3 k}^{*}(z)= & 2 \pi i \chi_{n}^{-2}(z)\left[\frac{3 z^{4}+2 z^{2}-1}{4 z^{3}}\right. \\
& \left.+\frac{\left(z^{2}+1\right)^{2}}{2 z^{2}} \sum_{j=0}^{2 n-1} \frac{z_{j}-\bar{z}_{j}}{\left(z-z_{j}\right)\left(z-\bar{z}_{j}\right)}\right]
\end{aligned}
$$


Again by residue theorem we have

$$
\begin{aligned}
I_{2 k}^{*}(z) & =2 \pi i \lim _{y \rightarrow z} \frac{2 y^{4}+4 y^{3} z+4 y z-2}{(y+z)^{3}} \\
& =2 \pi i\left(\frac{3 z^{4}+2 z^{2}-1}{4 z^{3}}\right) .
\end{aligned}
$$

By (5.33), (5.34), (5.35), (5.31) and (5.30) and taking into account that $\chi_{n}^{2}\left(y_{k}\right)=1$ it follows that

$$
I_{k}^{*}=-\frac{\pi i\left(1+y_{k}^{2}\right)^{4}}{32 y_{k}^{2}} \sum_{j=0}^{2 n-1} \frac{z_{j}-\bar{z}_{j}}{\left(y_{k}-z_{j}\right)\left(y_{k}-\bar{z}_{j}\right)} .
$$

Since, $y_{k}=\sqrt{\left(1-x_{k}\right) /\left(1+x_{k}\right)}$ and $z_{k}=i \sqrt{\left(1+a_{k}\right) /\left(1-a_{k}\right)}$, thus by simple calculation, we have,

$$
\begin{aligned}
\sum_{j=0}^{2 n-1} \frac{z_{j}-\bar{z}_{j}}{\left(y_{k}-z_{j}\right)\left(y_{k}-\bar{z}_{j}\right)} & =\sum_{j=0}^{2 n-1}\left(\frac{1}{y_{k}-z_{j}}-\frac{1}{y_{k}-\bar{z}_{j}}\right) \\
& =\sum_{j=0}^{2 n-1} \frac{i \sqrt{\left(1+a_{j}\right)} \sqrt{\left(1-a_{j}\right)}}{1+a_{j} x_{k}}\left(\frac{2}{1+y_{k}^{2}}\right) \\
& =\frac{4 i \lambda_{2 n}\left(x_{k}\right)}{\left(1+y_{k}^{2}\right)}
\end{aligned}
$$

thus

$$
I_{k}^{*}=\frac{\pi \lambda_{2 n}\left(x_{k}\right)\left(1+y_{k}^{2}\right)^{3}}{8 y_{k}^{2}}
$$

Therefore by (5.28), the lemma follows.

Lemma 5.3. For $A_{0}$, defined by (5.3), we have

$$
A_{0}=\left(\frac{\pi}{\lambda_{2 n}(1)}\right)
$$

Proof. By using the transformation (5.11), (5.12), (5.15) and (5.16), we have

$$
A_{0}=\frac{1}{4 U_{n}^{2}(1)} \int_{-\infty}^{\infty} \frac{1+y^{2}}{y^{2}} \sin ^{2} \phi_{2 n}(y) d y
$$

which, due to (5.20), can expressed as

$$
A_{0}=-\frac{1}{16 U_{n}^{2}(1)}\left(I_{1}-2 I_{2}+I_{3}\right)
$$

where

$$
\begin{gathered}
I_{1}=\int_{-\infty}^{\infty} \frac{1+y^{2}}{y^{2}} \chi_{n}^{2}(y) d y \\
I_{2}=\int_{-\infty}^{\infty} \frac{1+y^{2}}{y^{2}} d y
\end{gathered}
$$

and

$$
I_{3}=\int_{-\infty}^{\infty} \frac{1+y^{2}}{y^{2}} \chi_{n}^{-2}(y) d y
$$


Since for $I_{1}, y=0$ is the only singular point in the upper half plane. Thus by the residue theorem, we have

$$
\begin{aligned}
I_{1} & =2 \pi i \lim _{y \rightarrow 0} \frac{d}{d y}\left\{\left(1+y^{2}\right) \chi_{n}^{2}(y)\right\} \\
& =4 \pi i \sum_{j=0}^{2 n-1}\left(\frac{1}{\overline{z_{j}}}-\frac{1}{z_{j}}\right) .
\end{aligned}
$$

Similarly,

$$
I_{3}=4 \pi i \sum_{j=0}^{2 n-1}\left(\frac{1}{\overline{z_{j}}}-\frac{1}{z_{j}}\right) .
$$

The integrand of $I_{2}(z)$ has only singular point $y=0$ in the upper half plane. Thus by the residue theorem, we have

$$
I_{2}=2 \pi i \lim _{y \rightarrow 0} \frac{d}{d y}\left(1+y^{2}\right)=0 .
$$

Hence using (5.40), (5.40) and (5.41) in (5.38) we get

$$
A_{0}=-\frac{\pi i}{2 \lambda_{2 n}^{2}(1)} \sum_{j=0}^{2 n-1}\left(\frac{1}{\overline{z_{j}}}-\frac{1}{z_{j}}\right)
$$

and since

$$
\sum_{j=0}^{2 n-1}\left(\frac{1}{\overline{z_{j}}}-\frac{1}{z_{j}}\right)=-\frac{4}{i} \lambda_{2 n}(1)
$$

hence

$$
A_{0}=\frac{2 \pi}{\lambda_{2 n}(1)}
$$

which in turn proves the lemma.

By Lemma 5.2, Lemma 5.3 and (5.4), Theorem 5.1 follows.

Conflicts of Interest: The author(s) declare that there are no conflicts of interest regarding the publication of this paper.

\section{REFERENCES}

[1] P. Borwein and T. Erdélyi, Polynomials and Polynomial Inequalities, Graduate Texts in Mathematics 161, Springer-Verlag, New York (1995).

[2] A. L. Lukashov, Inequalities for the derivatives of rational functions on several intervals, Izv. Math. 68(3) (2004), 543-565.

[3] A. A. Markov(1951), Izbrannye trudy, Teoriya cisel. Teoriya veroyatnostei, Izdat. Akad. Nauk SSSR, Leningrad.

[4] G. Min, Lobatto-type quadrature formula in rational spaces, J. Comput. Appl. Math. 94(1) (1998), 1-12.

[5] Y. Rouba, K. Smatrytski and Y. Dirvuk, Rational quasi-Hermite-Fejer-type interpolation and Lobatto-type quadrature formula with Chebyshev-Markov nodes, Jaen J. Approx. 7(2) (2015), 291-308

[6] E. A. Rovba, Interpolation rational operators of Fejér and de la Valle-Poussin type, Mat. Zametki., 53(2) (1993), 114-121 (in Russian, English translation: Math. Notes. 53 (1993), 195-200. 
[7] E. A. Rouba, Interpoljacija i rjady Furie v ratsionalnoj approksimatsii, GrSU, Grodno. (2001).

[8] Y. A. Rouba and K. A. Smatrytski, Rational interpolation in the zeros of Chebyshev-Markov sine-fractions, Dokl. Nats. Akad. Nauk Belarusi, 52(5) (2008), 11-15 (in Russian).

[9] V. N. Rusak, Interpolation by rational functions with fixed poles, Dokl. Akad. Nauk BSSR 6 (1962), 548-550 (in Russian).

[10] V. N. Rusak, On approximations by rational fractions, Dokl. Akad. Nauk BSSR 8 (1964), 432-435 (in Russian).

[11] A. H. Turecki, Teorija interpolirovanija v zadachakh, Izdat "Vyssh. Skola", Minsk. (1968).

[12] J. Van Deun, Electrostatics and ghost poles in near best fixed pole rational interpolation, Electron. Trans. Numer. Anal. 26 (2007), 439-452. 